\title{
Relación entre la composición química inorgánica del agua, la precipitación y la evaporación en la cuenca de Río Grande, Chone, Ecuador
}

\section{(Relationship between the inorganic chemical composition of water, precipitation and evaporation in the basin of Rio Grande, Chone, Ecuador)}

\author{
David Carrera ${ }^{1}$, Tania Crisanto ${ }^{1}$, Paulina Guevara ${ }^{1}$, Maribel Maya ${ }^{2}$
}

\begin{abstract}
Resumen:
En la cuenca de Río Grande se construye la presa Propósito Múltiple Chone (PMCH) con una inversión aproximada de 66 millones de dólares que irrigará más de 7000 ha. Por las marcadas diferencias en las precipitaciones, éstas podrían perjudicar la calidad de las aguas, por consiguiente, el objetivo de este trabajo fue establecer la relación existente entre los iones y su ubicación en el gráfico: relación de los iones y mecanismos de procesos químicos de las aguas estableciendo su comportamiento y tendencia. El trabajo fue una investigación no experimental, transversal y descriptiva. Se tomaron 111 muestras simples en el conjunto de cauces que forman la cuenca de Río Grande, en los años 2013 y 2014, durante las épocas de estiaje y lluvia. Los parámetros medidos en las muestras fueron aniones y cationes. Los procesos que controlan la química del agua superficial de la zona de estudio en época de lluvia poseen una predisposición a la mineralización en equilibrio con las rocas, sin embargo, en el periodo de estiaje se observó la predisposición de las aguas hacia la zona donde predomina la evaporación con respecto a la precipitación, aumentando la composición química inorgánica de las aguas que en el tiempo podría ampliarse.
\end{abstract}

Palabras clave: procesos químicos; mineralización; bicarbonatos; calcio

\begin{abstract}
:
In the Rio Grande basin, the Chone Multi-Purpose dam (PMCH) is built with an investment of approximately $\$ 66$ million, to irrigate over 7000 ha. The marked differences in precipitation could impair the quality of water; therefore the aim of this study was to establish the relationship between the ions and their location in the graph: relationship of ions and mechanisms of chemical processes, water behavior and trend setting. The study was a non-experimental, cross-sectional and descriptive research. 111 samples were collected in the group of channels that form the Rio Grande basin, in 2013 and 2014 during times of drought and rain. The parameters were measured in the sample anions and cations. The processes that control the chemistry of surface water in the studied area during the rainy season have a predisposition to mineralization in equilibrium with rocks. However, in the time of drought, water movement was observed towards the area where evaporation with respect to precipitation predominates, increasing the inorganic chemistry of the waters that, in time, could be extended.
\end{abstract}

Keywords: chemical processes; mineralization; bicarbonates; calcium

\footnotetext{
${ }^{1}$ Universidad de las Fuerzas Armadas, Departamento de Ciencias de la Tierra y la Construcción, Sangolquí - Ecuador (dvcarrera@espe.edu.ec, ttcrisanto@espe.edu.ec, pvguevara@espe.edu.ec )

${ }_{2}^{2}$ Universidad de las Fuerzas Armadas, Departamento de Ciencias Económicas y AdministrativasEcuador (ammaya@espe.edu.ec )
} 


\section{Introducción}

La provincia de Manabí, al oeste del Ecuador, tiene gran variabilidad en la cantidad de pluviosidad que recibe anualmente. En un período de 20 años el Instituto Nacional de Meteorología e Hidrología (INAMHI), en su estación ubicada en Chone, registró en el año 1998 un valor aproximado a 2500 mm, en tanto que, en el año 1996 el valor fue próximo a $600 \mathrm{~mm}$, con un promedio anual cercano a los de $1200 \mathrm{~mm}$, lo que dificulta la distribución permanente de la demanda de agua por parte de los habitantes y la biota en general. El Estado ecuatoriano, con el objetivo de evitar excesos o carencias del recuso hídrico en la zona, ejecuta el Proyecto Multipropósito Chone $(\mathrm{PMCH})$ que tiene como objetivos la construcción de la presa de Río Grande y la construcción de un sistema de riego para abastecer una demanda de aproximadamente 7000 ha.

De acuerdo al Estudio de Impacto Ambiental realizado para la construcción del proyecto Multipropósito Chone, difundido por la Secretaría Nacional del Agua (SENAGUA), en diciembre de 2010 se tomó datos de la estación M162 Chone durante el segundo semestre del año 2004 y se demostró un déficit hídrico de entre 200 a 600 mm debido a las bajas precipitaciones (Figura 1).

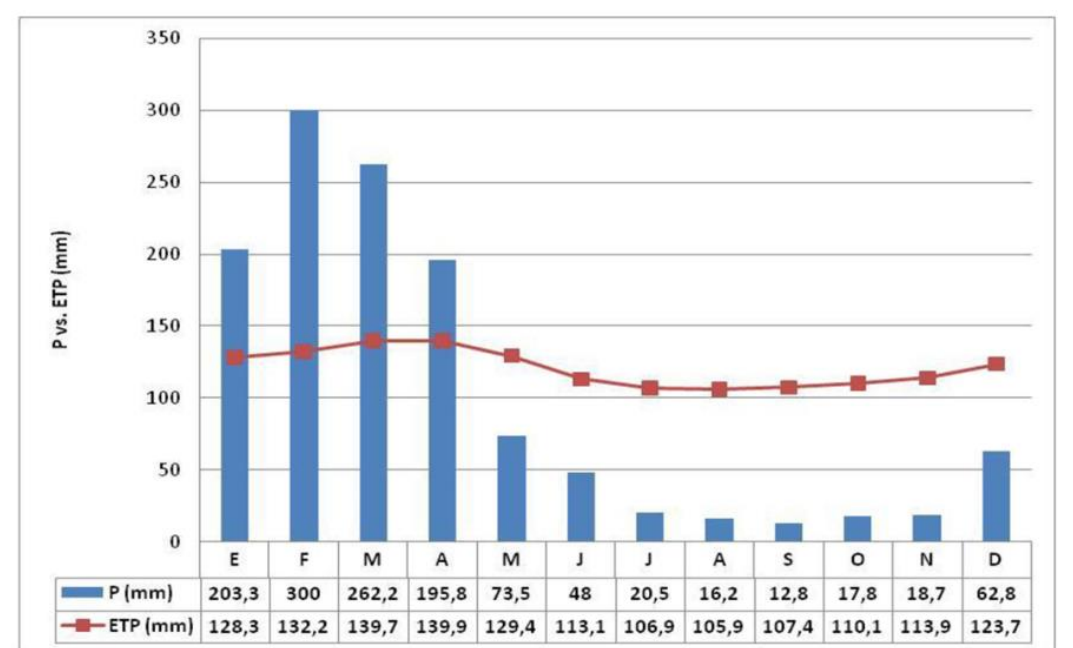

Figura 1. Climograma del año 2004 con datos de la estación M162 Chone Fuente. SENAGUA, 2010

Por las marcadas diferencias en las precipitaciones, el objetivo del presente trabajo fue establecer la relación existente entre la composición química inorgánica del agua, la precipitación y la evaporación en la cuenca de Río Grande y ubicarla en el gráfico: relación de los iones y mecanismos de procesos químicos de las aguas (Gibbs, 1970) 


\section{Materiales y Métodos}

El presente trabajo fue una investigación no experimental, transversal y descriptiva (Díaz, 2009) con muestreo en el 100\% de los cuerpos de agua (Guayacán, Juan Cayo, la Balsa, Coñaque, Sánchez y Platanales) que van a drenar a la cuenca del Río Grande.

Con el fin de observar el incremento en la composición química inorgánica del agua, se muestreó en el nacimiento, tramo medio y desembocadura al cauce principal de la cuenca, tal como se indica en la Figura 2.

Se tomaron 71 muestras simples en la época lluviosa (enero y febrero de 2013) y 40 similares, en época de estiaje (agosto y septiembre 2014) en toda la cuenca del Río Grande con el objetivo de comparar el incremento-decremento de la composición química inorgánica del agua en relación con la precipitación y la evaporación.

La diferencia del número de muestras de la época lluviosa a la de estiaje radicó en que debido a la anegación de la cuenca no se pudo acceder a todos los puntos tomados en época de estiaje, sin embargo, los puntos neurálgicos fueron tomados, tal es el caso del punto donde se construye la presa $\mathrm{PMCH}$, que corresponde en el estiaje al punto 24 y en Iluvias al punto 11

Mediante datos obtenidos de la estación M162 del INAMHI se determinó las variaciones anuales y mensuales de precipitación durante un período de 20 años, datos que fueron completados y validados anticipadamente con apoyo de la estación Portoviejo, y se comprobó estadísticamente la información de acuerdo a lo que propone Monsalve (2009).

Los parámetros medidos durante el muestreo fueron: cationes de sodio, potasio, calcio, magnesio; y aniones de carbonatos, bicarbonatos, sulfatos y cloruros que fueron analizados en el laboratorio LASA, el cual está acreditado al OAE, Organismo de Acreditación Ecuatoriano (http://www.laboratoriolasa.com/web/pags/cuerpo.html)

En el litoral ecuatoriano existe un régimen de tipo tropical con una estación lluviosa única entre Diciembre y Abril (Pourrut, 1995) lo que se observa en la Figura 3.

Con los resultados obtenidos se elaboraron curvas de distribución de los diferentes aniones y cationes de acuerdo al sistema hidrográfico, la altitud y los diferentes períodos, para observar cuál es la distribución de la concentración inorgánica de las aguas. Además, para comprender las variaciones producidas por las marcadas condiciones climáticas se empleó la curva que controla los procesos químicos del agua superficial propuesta por Gibbs (1970) y que sigue vigente tal como lo demostró Yu et al. (2014). 


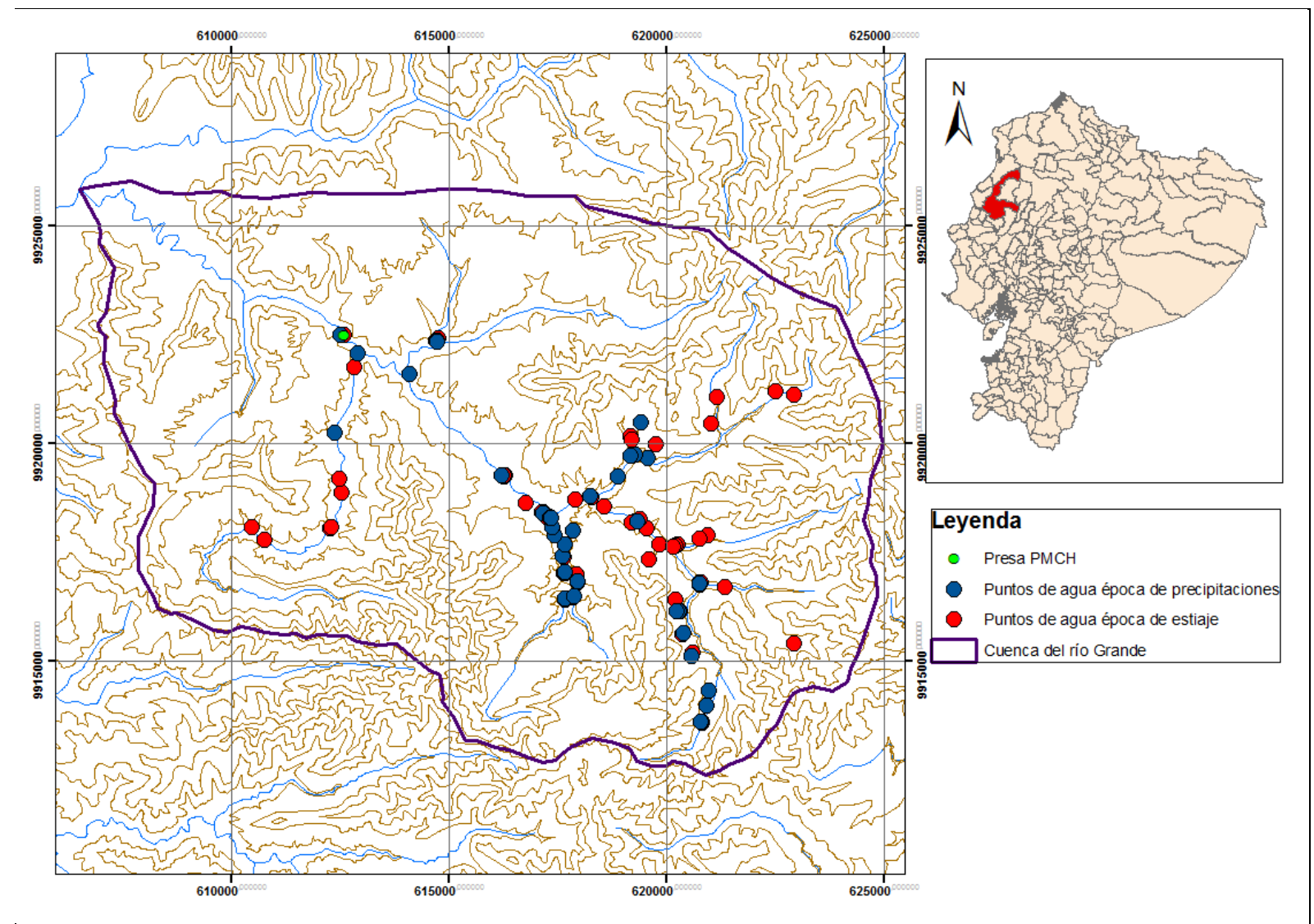

Figura 2. Ubicación de los puntos de agua muestreados

\section{Resultados}

\section{Precipitación}

Antes de determinar las variaciones mensual y anual de las precipitaciones los datos de la estación M162 de Chone fueron completados y verificados estadísticamente, para ello se utilizó también la estación M005 de Portoviejo. La variación en la precipitación promedio mensual se observa en la Figura 3 y la variación promedio anual se observa en la Figura 4.

La Figura 4 demuestra que las precipitaciones promedio máximas están en los años 1997, 1998 y 1999 siendo cercanas a los 2000 mm, en cuanto a las precipitaciones mínimas anuales fueron en los años 1991 y 1996 con valores cercanos a los 600mm.

La cuenca hidrográfica del Río Grande, donde se construye el $\mathrm{PMCH}$ tiene un comportamiento hidrológico variado, desde precipitaciones que superan los $2000 \mathrm{~mm}$ que aguas abajo generan inundaciones y pérdidas económicas, hasta precipitaciones con valores inferiores a los $600 \mathrm{~mm}$ ocasionando pérdidas en los campos de cultivo por falta de agua para riego 


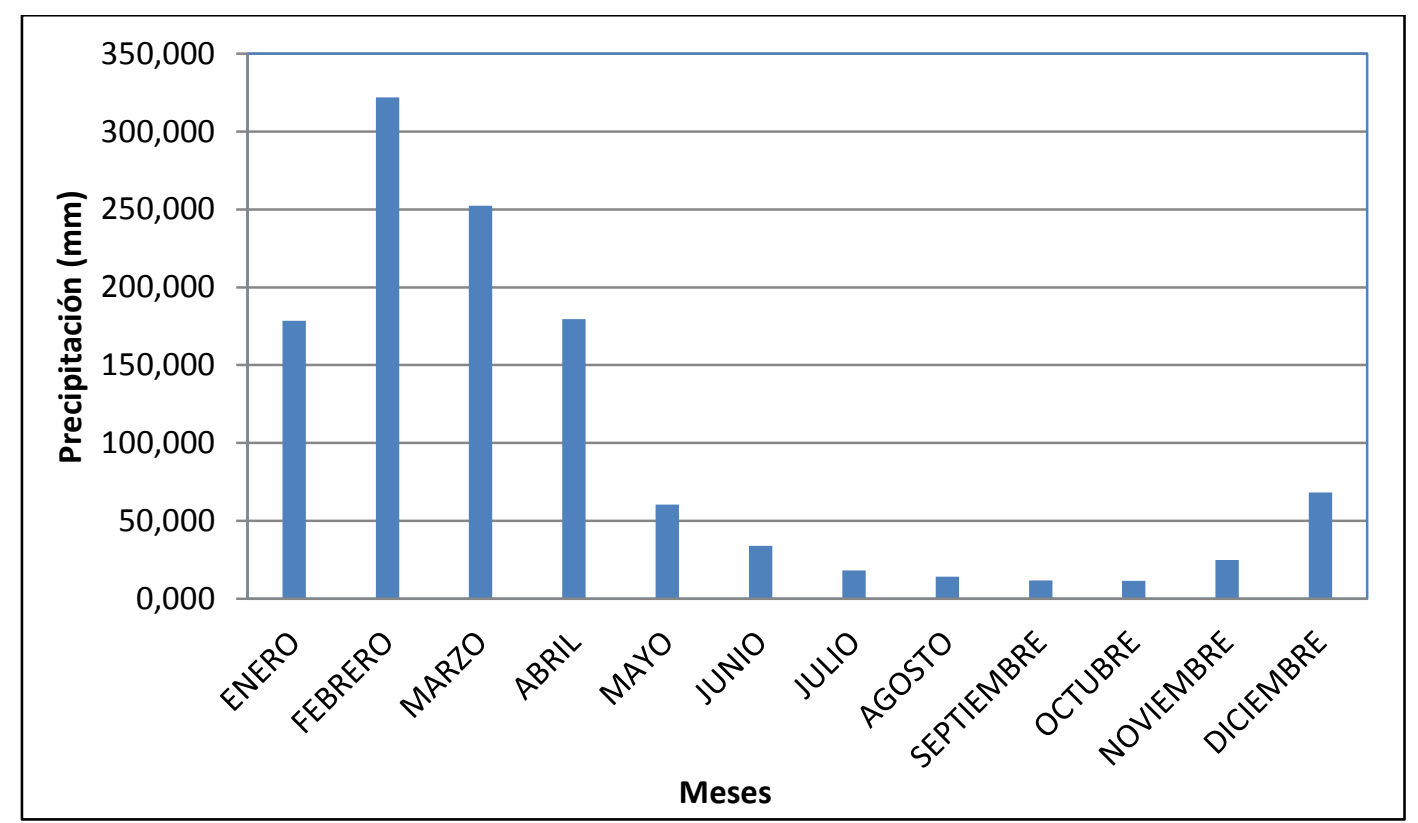

Figura 3. Variación de la precipitación mensual en 20 años en la estación Chone

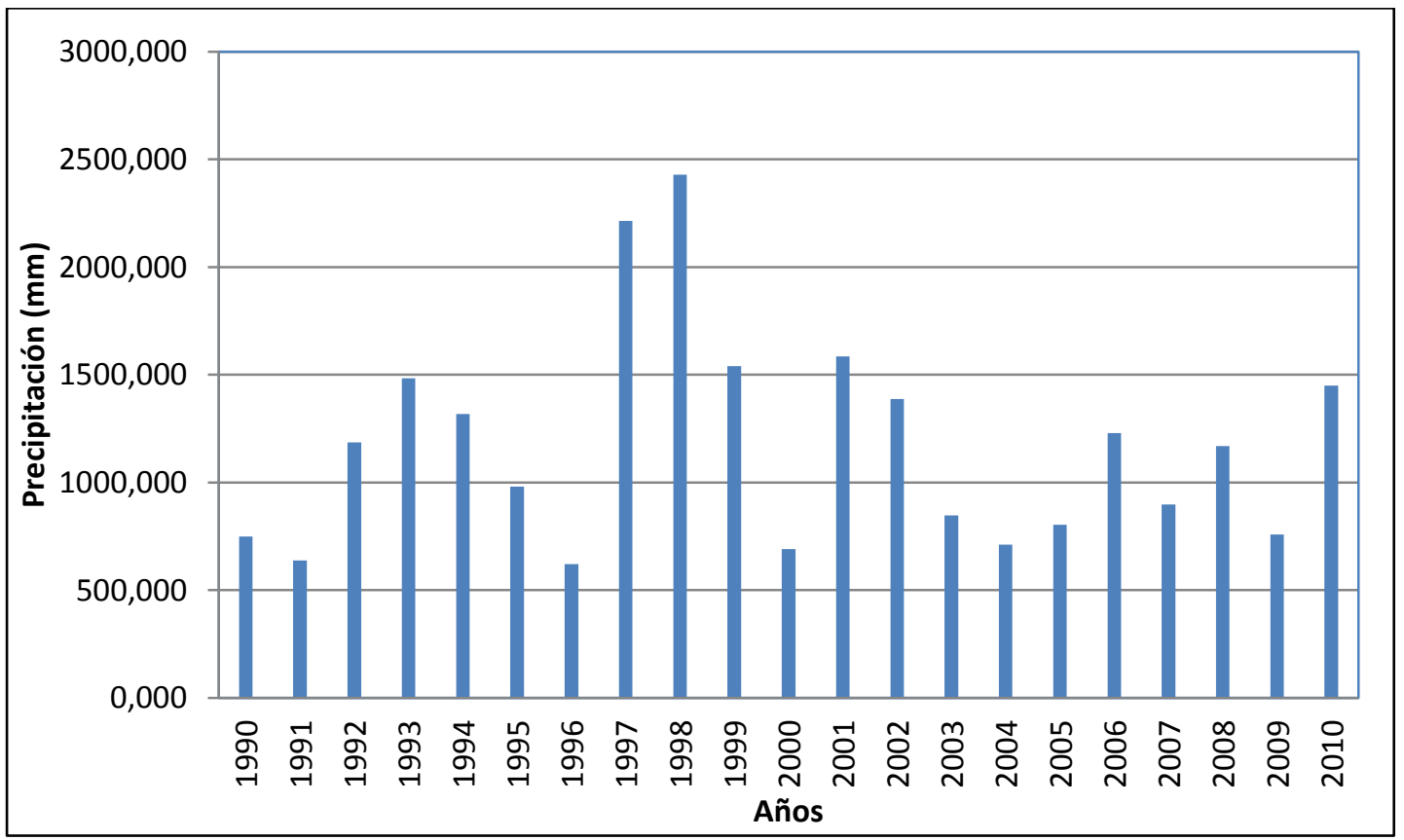

Figura 4. Variación de la precipitación anual en 20 años en la estación Chone

\section{Discusión}

El promedio de la concentración de aniones y cationes en la época de lluvias fue de 175 $\mathrm{mgL}^{-1}$, mientras que en la época de estiaje fue de $240 \mathrm{mgL}^{-1}$, que inicialmente es lógico pues en el primer caso están diluidas las especies químicas y en el segundo concentradas, sin embargo, esto no siempre es una regla como lo citó Carrera y Pérez (2013), en la cuenca del río Milagro.

Existe una predominancia del anión bicarbonato, que probablemente su contenido se origine en el desgaste y disolución de rocas en la cuenca que contienen carbonatos tales como la 
piedra caliza, definida por la geología de la zona (Carta Geológica de Chone MIII-F2 3591-I, 1979).

El catión predominante es el calcio, cuyo origen eventualmente se deba a la presencia de piedra caliza que presenta una baja solubilidad en agua pura; su disolución es promovida por la presencia de $\mathrm{CO}_{2}$ disuelto en el agua, el atmosférico o el generado en sedimentos ricos en materia orgánica (Fuentes y Masoll, 2002). En cuanto a la superioridad del calcio en ciertas zonas, posiblemente se deba a que los minerales predominantes son los feldespatos, además la geología del lugar describe que la zona de estudio posee restos fósiles, los que tienen como componente principal al calcio.

En la Figura 5 se presentan la distribución de aniones y cationes en los diferentes sistemas hidrográficos que conforman la cuenca del Río Grande para la época de estiaje, donde se ve que el mayor contenido de sales está en el punto 24 perteneciente al Río Grande, debido a que es la descarga de todos los afluentes secundarios. La sal predominante es el sulfato de calcio.

En la Figura 6 se presentan la distribución de aniones y cationes en los diferentes sistemas hidrográficos que conforman la cuenca del Río Grande para la época de lluvias, donde se ve que el mayor contenido de sales está en el punto 11 perteneciente al Río Grande, donde se construye la PMCH. La sal predominante es el bicarbonato de calcio.

\section{Diagrama que representa los procesos que controlan la química de las aguas superficiales}

Gibbs (1970) identificó tres mecanismos naturales que controlan la química del mundo de las aguas continentales superficiales: la mineralización en equilibrio con las rocas, predominancia de la evaporación con respecto a la precipitación y predominancia de la precipitación con respecto a la evaporación. Para definir el comportamiento de la cuenca del Río Grande se agrupó las estaciones por sistemas hidrográficos, resultando seis en la época de lluvias y siete en periodo de estiaje. De cada uno de los sistemas se obtuvo el valor promedio de los sólidos totales disueltos y de la relación $\mathrm{Na}(\mathrm{Na}+\mathrm{Ca})$ (Figura 7).

Los resultados indican que las aguas del periodo de estiaje presentan una predisposición a la concentración del tipo bicarbonatada cálcica, ubicándose los puntos con tendencia a la zona de predominancia de la evaporación con respecto a la precipitación. Este tipo de zonas típicamente se ubican en el continente africano y asiático donde las lluvias son escasas y por tanto los problemas de sequía son comunes (Gibbs, 1970) 


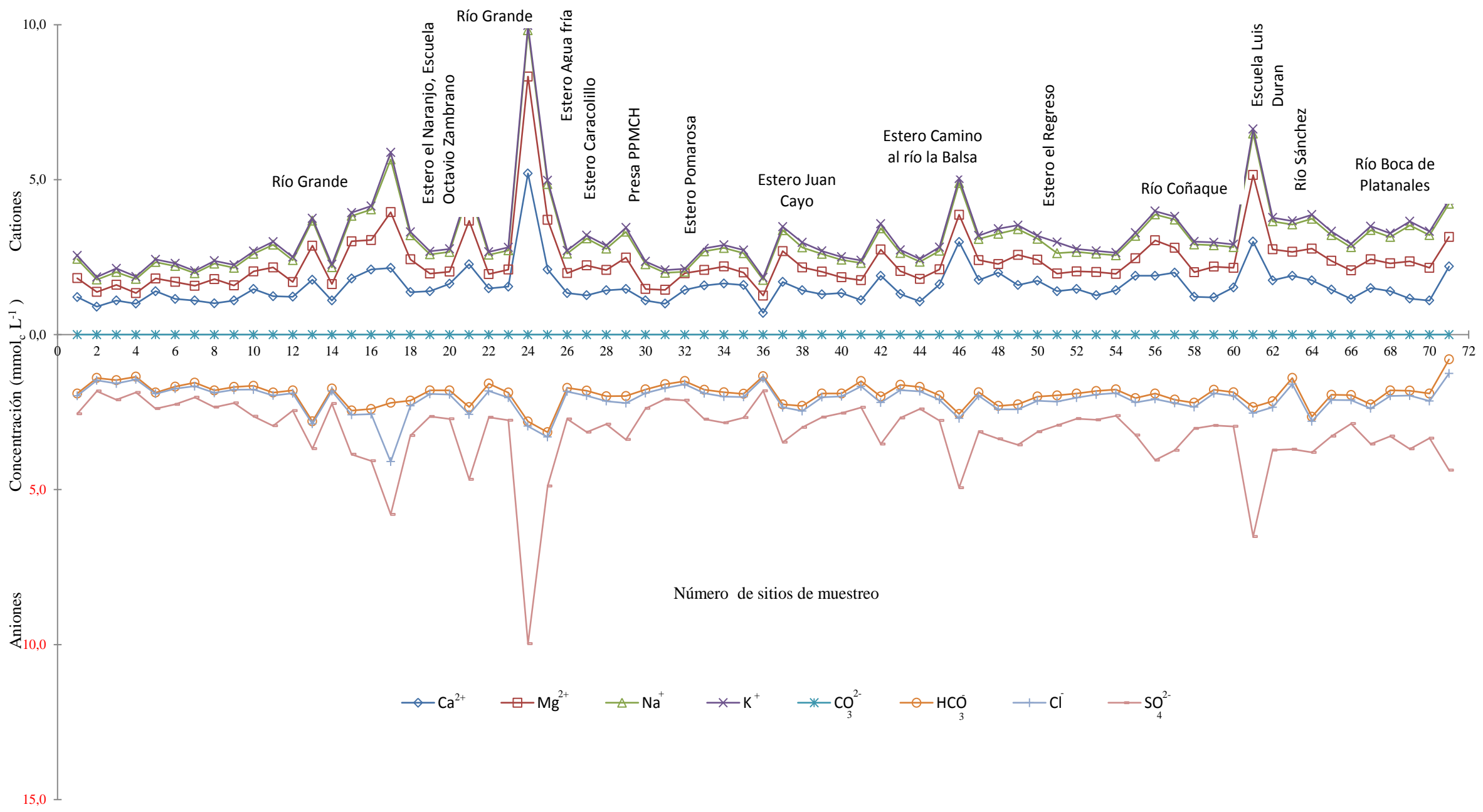

Figura 5. Distribución de iones del muestreo de aguas en la cuenca donde se construye el PMCH (estiaje)

Enfoque UTE, V.6-N.1, Mar.2015, pp.25 - 34 


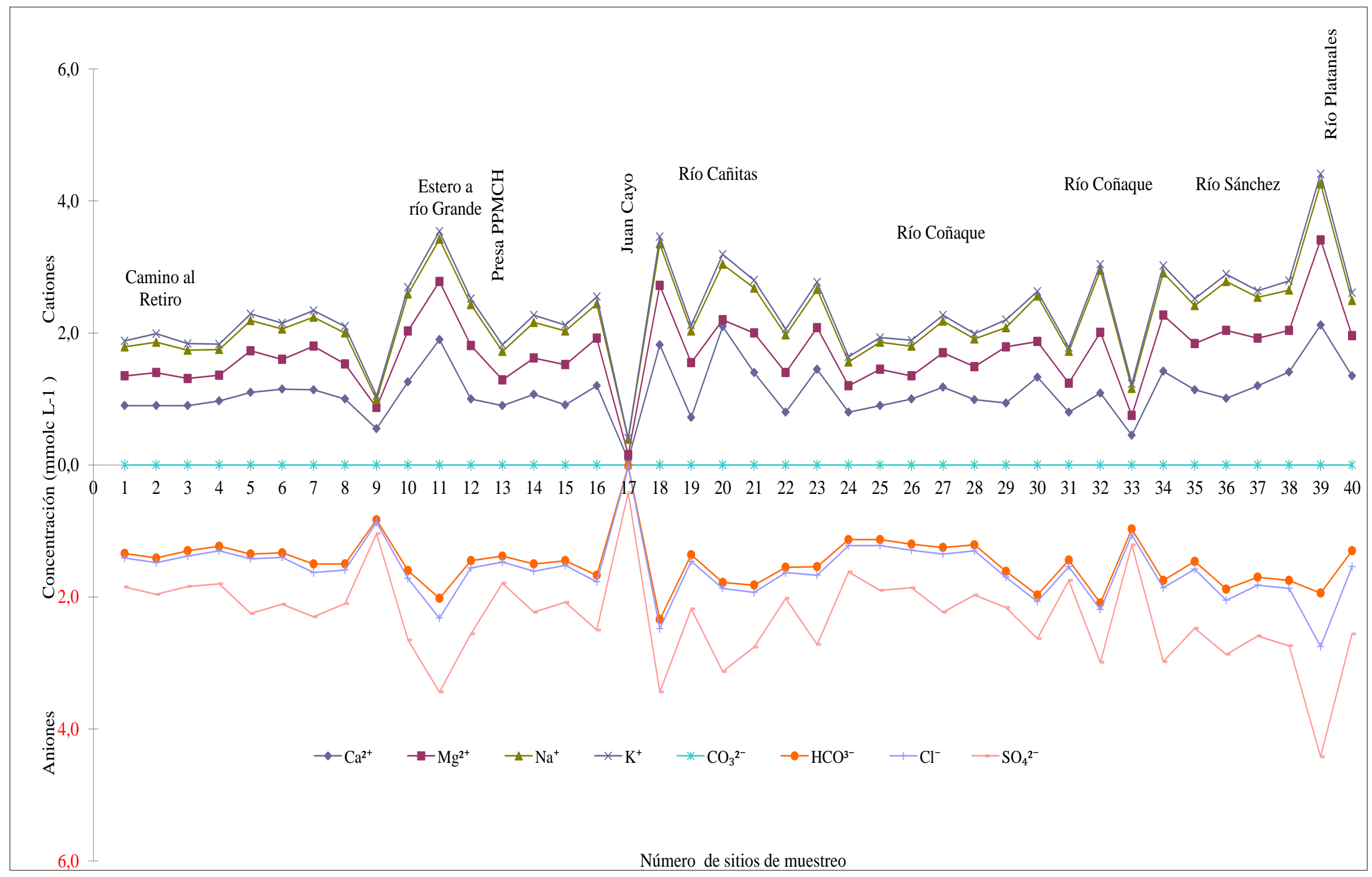

Figura 6. Distribución de iones del muestreo de aguas en la cuenca donde se construye el PMCH (época de lluvia). 


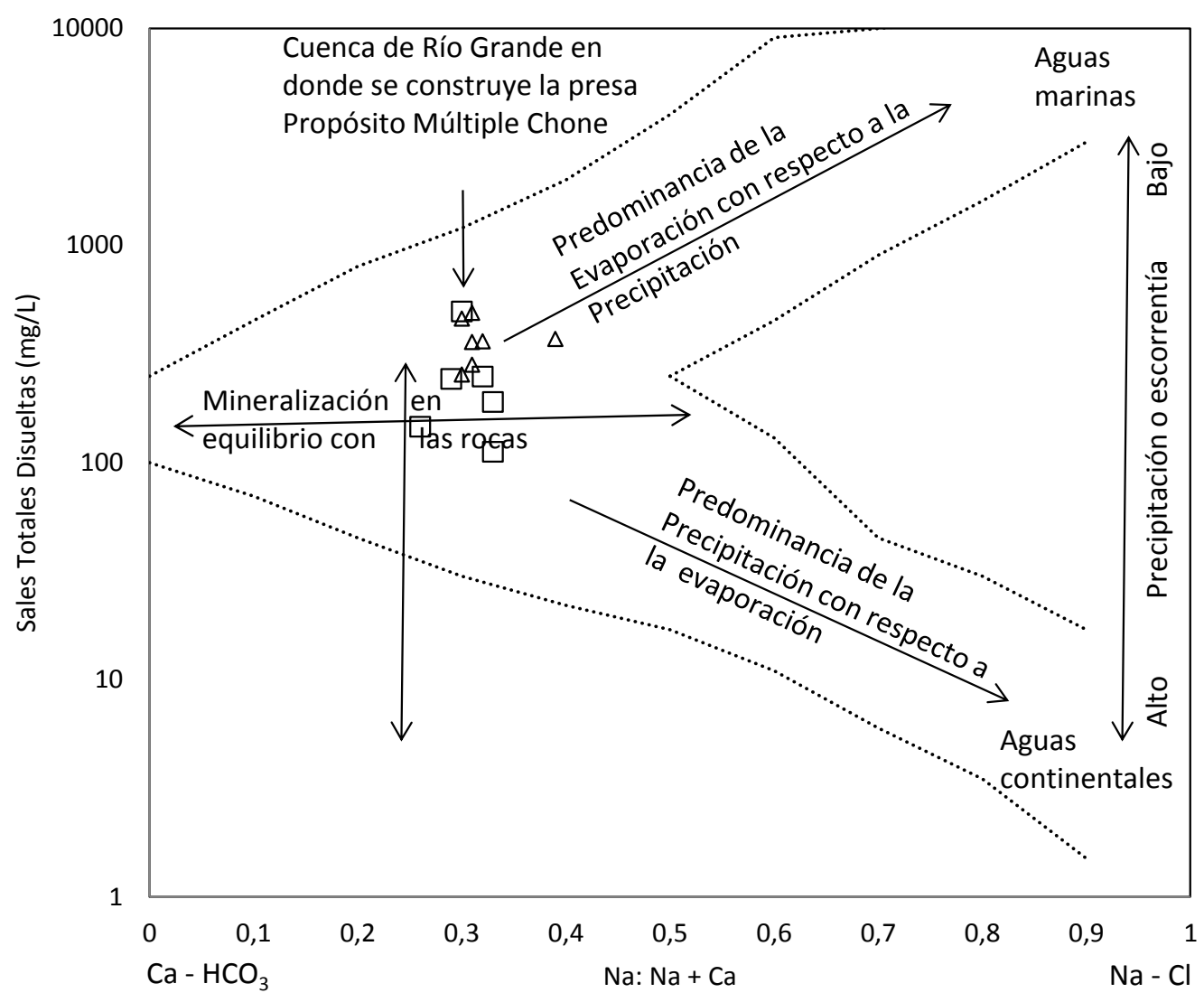

Figura 7. Puntos de agua de la época de estiaje $(\Delta)$ y de lluvia ( $\square)$, ubicados en la curva de salinidad de Gibbs.

En cuanto a las aguas en el período de lluvia presentan una predisposición a la concentración del tipo bicarbonatada cálcica, ubicándose los puntos con tendencia a la zona de mineralización en equilibrio con las rocas. Este tipo de zonas típicamente se ubican en trópico de Sudamérica, con características de suelos pobres por el lavado de minerales (Carrera et al., 2011)

La precipitación se está reduciendo y la composición química inorgánica de las aguas de Río Grande aumentando en la época de estiaje que corresponde a ocho meses en el año, por lo que es necesario establecer un plan de manejo ecosistémico de la cuenca hidrográfica en donde se construye el PMCH.

Se debe considerar el caso de la cuenca de Río Grande, que se constituye en un caso atípico pues en la zona ecuatorial donde está ubicado el Ecuador las precipitaciones son altas en comparación con el resto del planeta (Aparicio, 2012)

\section{Conclusiones y Recomendaciones}

La cuenca hidrográfica de Río Grande, en donde se construye el PMCH, presentó una irregular distribución de lluvias en un período de 20 años. En el fenómeno del niño las precipitaciones superaron los $2500 \mathrm{~mm}$ generando inundaciones, mientras que en otros 
años, existió un estiaje con precipitaciones que no alcanzaron los $600 \mathrm{~mm}$ al año. Los procesos que controlan la química del agua superficial de los sistemas hidrográficos que forman Río Grande poseen una predisposición a la zona de mineralización en equilibrio con las rocas, sin embargo, en el periodo de estiaje se observó la tendencia hacia la zona donde predomina la evaporación con respecto a la precipitación aumentando la composición química inorgánica de las aguas de la cuenca del Rio Grande en Chone-Ecuador, que en el tiempo podría ampliarse.

Se recomienda la realización de un muestreo sistemático en los puntos analizados.

\section{Bibliografía}

Aparicio, F. (2012) Fundamentos de hidrología de superficie, Limusa Impreso en México. p 289

Carrera, D. Pérez A. (2013). Salinidad, fosfatos, nitratos y problemas de infiltración en las aguas de irrigación de Milagro. Revista Ciencia UNEMI p 83-95

Carta Geológica de Chone MIII-F2 3591-I. (1979).

Díaz, V. Metodología de la investigación científica y bioestadística. Ediciones. RIL editores. $p$ 121-122

Fuentes, F., \& Massol-Deya, A. (2002). Manual de laboratorio, ecología de microorganismos. Puerto Rico.

Gibbs, R.J. (1970) Mechanisms controlling world water chemistry, Science, 170: 1088-1090.

INAMHI. (2008). Estudio Hidrológico de Inundaciones en la Cuenca Alta del Río Chone. Quito, Ecuador.

LABORATORIA LASA. Disponible en http://www.laboratoriolasa.com/web/pags/cuerpo.html

Monsalve, G. (2009). Hidrología en la Ingeniería. (1ra ed. 3ra Reimp.) Alfaomega, p 358

Pourrut, P. (1995). El agua en el Ecuador: Clima, precipitaciones escorrentía. Corporación editora Nacional. p. 118

Secretaria Nacional del Agua SENAGUA. (2012)., Ecuador. Construcción del Puente San Pablo en el canal San Antonio. Chone.

Secretaria Nacional del Agua, Ecuador (2010) Proyecto Propósito Múltiple Chone. Estudio de Impacto Ambiental y Plan de Manejo Ambiental, Capitulo 5

Yu, Y., Song, X., Zhang, Y., Zheng, F., Liang, J., Liu L., (2014). Identifying spatio-temporal variation and controlling factors of chemistry in groundwater and river water recharged by reclaimed water at Huai River, North China. Stoch Environ. Res. Risk. Asses. 28:1135-114 